\title{
X-ray study of dopant state in highly doped semiconductor single crystals
}

\author{
I.L. Shul'pina ${ }^{1}$, R.N. Kyutt ${ }^{1}$, V.V. Ratnikov ${ }^{1}$, I.A. Prokhorov ${ }^{2}$, I.Zh. Bezbakh ${ }^{2}$, M.P. Shcheglov ${ }^{1}$ \\ ${ }^{I}$ Ioffe Physico-Technical Institute RAS, \\ Politekhnicheskaya 26, 194021 St.-Petersburg, Russia \\ ${ }^{2}$ Space Materials Science Research Center of the Crystallography Institute RAS, \\ Akademicheskaya 8, 248640 Kaluga, Russia \\ E-mail: iren.shulpina@mail.ioffe.ru
}

\begin{abstract}
Using $\mathrm{Si}(\mathrm{As}, \mathrm{P}, \mathrm{B})$ and $\mathrm{GaSb}(\mathrm{Si})$ study, possibilities of X-ray diffraction methods for diagnostics of highly doped semiconductor crystals in characterization of dopant state - whether it is in the crystals in the form of solid solution or under various stages of its decomposition - are shown. The combination of techniques of X-ray diffraction topography and high resolution X-ray diffractometry, higher sensitive to the crystal lattice strain than that traditionally used is taken as the basis for investigating the crystals with slight and strong absorption of X-rays. These methods were supplemented with digital processing of the topographic images of growth striations and electrical measurements.
\end{abstract}

Keywords: microdefects, clusters, growth striations, X-ray topography, X-ray diffractometry.

Manuscript received 25.01.10; accepted for publication 02.12.10; published online 28.02.11.

\section{Introduction}

Highly doped semiconductor crystals are widely used in solid-state microelectronics when various electronic devices are fabricated [1]. In particular, silicon single crystals with a high doping concentration are used in power electronics as the substrates for epitaxial structures. In this case, it is important the combination of large-square plates with high structural perfection and low resistivity [2]. The latter denotes a high concentration of impurities. In these crystals, the initial stage of decomposition of dopant solid solution is possible already during crystal growth in the form of generation of point defect clusters. Under subsequent heat treatments, this process develops with the formation of secondary defects in the form of precipitations and dislocation loops. These defects influence very much on properties of devices.

Highly doped semiconductor single crystals of $A_{3} B_{5}$ compounds are convenient model materials used to study features of crystallization processes under microgravity conditions on-board space vehicles. Using them makes it possible to enhance the sensibility of response of crystal structures to perturbations of the crystallization process $[3,4]$.

The diffraction methods - X-ray topography, high resolution X-ray diffractometry and electron microscopy - are effective for characterization of real structure of the crystals. These methods were applied successfully for the study of doped crystals after postgrowth heat treatment when generating defects in them were well pronounced [5-8]. In the as-grown state, when there are a few defects or they have a very fine size and weakly deform crystal lattice, the standard diffraction methods are not sufficiently sensitive. In this work, the experience of application of more sensitive, based on interference effects of X-ray diffraction for diagnostics of highly doped crystals as well as after heat treatment in the as-grown state $[2,9,10]$ is described.

In the study of crystals by using the X-ray diffraction methods, the level of X-ray absorption is very important, because it defines the thickness of information layer and the features of defect revealing. Therefore, it is appropriate to consider separately the cases of low and high absorption. With this viewpoint, silicon is the most typified by the crystals with low $\mathrm{X}$ - 
ray absorption. GaSb was selected as the crystal with high absorption.

\section{Studied samples and methods of research}

In $[2,9,10]$ the silicon single crystals $150 \mathrm{~mm}$ in diameter grown by Czochralski and doped by As, $\mathrm{P}$ and $\mathrm{B}$ separately during the growth were studied. Concentration of dopants was $(2.5-4.0) \times 10^{19} \mathrm{~cm}^{-3}$, $1.1 \times 10^{20} \mathrm{~cm}^{-3}$ and $(4-6.5) \times 10^{18} \mathrm{~cm}^{-3}$, respectively. They were grown at the Technological Center of Sumimoto Mitsubishi Corporation (USA) using the method allowing for monotonous decrease in the oxygen concentration in the direction from a seed end to the tail end of the crystal ingot within $(1.5-0.79) \times 10^{19} \mathrm{~cm}^{-3}$. The ingots were sliced to prepare $0.75 \mathrm{~mm}$ thick round plates at different axial positions. The plates have been chemically etched to remove the residual damage prior to cut in samples corresponding to specific position along the plate diameter.

After study of the samples in as-grown state, the series of the crystals highly doped with boron was investigated as well in the as-grown state after two standard conditions of ASTM heat treatment. Two standard conditions of ASTM heat treatment, A and C, were compared which differ by the additional stage of low-temperature nucleation in C: $T=1050{ }^{\circ} \mathrm{C}$ for $16 \mathrm{~h}$ in an oxygen atmosphere with $5 \%$ nitrogen (A) and slow annealing up to $T=800{ }^{\circ} \mathrm{C}$ for $4 \mathrm{~h}$, plus $1050{ }^{\circ} \mathrm{C}$ for $16 \mathrm{~h}$ in an oxygen atmosphere with $5 \%$ nitrogen $(\mathrm{C}$, modified B mode).

As a strongly absorbing medium, two GaSb single crystals doped with $\mathrm{Si}$ were studied. Initial GaSb single crystal with a diameter of $35 \mathrm{~mm}$ was grown in the [111] direction by the Czochralski method and doped with $\mathrm{Si}$ during the growth process up to the concentration of approximately $2 \times 10^{19} \mathrm{~cm}^{-3}$. Two cylindrical crystals with diameters $23 \mathrm{~mm}$ and lengths $75 \mathrm{~mm}$ were cut from the obtained ingot in the direction of growth. These crystals were partially regrown under identical thermal conditions by the vertical Bridgman method, and the seed was located in the lower, "cold" part of the setup. The intensity of natural convection was decreased considerably in this case. The specific features of crystal growth were considered in more detail in [4].

The crystals (by convention, we let the letters $\mathrm{K}$ and $\mathrm{N}$ to denote them) were obtained as result and had parts with considerably different structures. Growth striations were a dominant type of defects in one of them (the seed grown by the Czochralski method and having the dislocation density $N_{D} \sim 10^{2} \mathrm{~cm}^{-2}$ ), and dislocations in the other part (the regrown part with the dislocation density $N_{D} \sim 10^{4} \mathrm{~cm}^{-2}$ ). For close concentrations of the dopant in both parts of the crystal (the equilibrium distribution coefficient of $\mathrm{Si}$ in $\mathrm{GaSb}$ is close to unity), this make it possible to analyze separately contributions of the formed defects (the growth striations and dislocations) to the crystal heterogeneity. Samples to be studied were the plates with the thickness $450 \mu \mathrm{m}$, and they were cut along the crystal's growth axis (along the (110) plane).

At first, all the specimens were studied with traditional techniques of X-ray topography (projection Lang method in $\mathrm{MoK}_{\alpha}$ radiation and back reflection Bragg method in $\mathrm{CuK}_{\alpha}$ and $\mathrm{MoK}_{\alpha}$ radiation) [11] to make an estimate of its real structure. The study was supplemented with a precious measurement of lattice parameters after [12]. Afterwards, more sensitive techniques to distortions of a crystal lattice based on the interference X-ray diffraction effects were attracted section Lang method and X-ray diffractometry under conditions of the Borrmann effect. The anomalous X-ray transmission method was used for investigation of $\mathrm{GaSb}(\mathrm{Si})$ single crystals with the digital processing the growth striation images and X-ray diffractometry methods in combination with some electrical measurements (Hall and spreading resistance methods).

\section{Experimental results}

\section{Study of silicon crystal in the as-grown state}

The investigation of silicon crystals doped with arsenic to $(2.5-4.0) \times 10^{19} \mathrm{~cm}^{-3}$ using the conventional X-ray projection topography techniques (the Lang method, anomalous X-ray transmission, Bragg diffraction) did not reveal the presence of dislocations and/or any other defects, although the background of topographs was sometimes different from the typical ones for perfect silicon crystals. The usual XRD measurements exhibited narrow rocking curves characteristic of perfect crystals and showed the absence of diffuse scattering. The crystal lattice parameter measurements pointed to possible macroscopic changes in lattice deformation below $10^{-5}$. Since the atomic radii of $\mathrm{Si}$ and $\mathrm{As}$ are close (1.17 and $1.126 \AA$, respectively), it was possible to assume more or less homogeneous distribution of dopant substituting silicon. Therefore, more sensitive techniques were attracted for the study of these crystals. Among X-ray topographic methods this is the section Lang method. It is known that it is more sensitive to the microdefects in silicon approximately by 20 times than the projection Lang method [7].

The silicon samples in the shape of $1.2-0.7 \mathrm{~mm}$ thick (100) oriented plates doped with arsenic without post-growth heat treatment, in as-grown state were studied. Section topographs were obtained in $\mathrm{MoK}_{\alpha}$ radiation, 422 and 440 reflections. These reflections were used for observation of important details of the topographs structure. However, it was required a long exposure about $20 \mathrm{~h}$ for fixation these topographs on photoplates for the nuclear investigation of $\mathrm{MK}$ emulsion type (X-ray tube BSV-29 type, $42 \mathrm{kV}, 20 \mathrm{~mA}$ ). For comparison, the projection topographs were obtained in the same reflections corresponding to position of section topographs. 


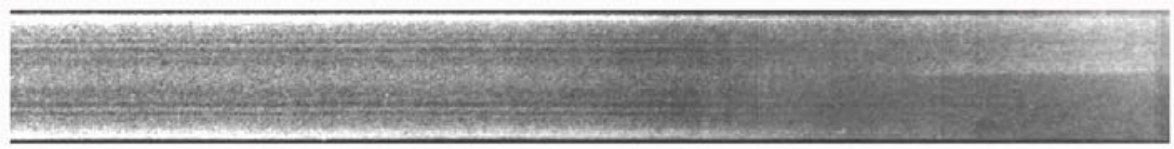

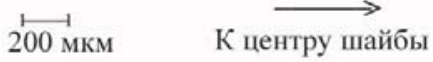

Fig. 1. A fragment of X-ray section topograph showing a missing of pendulum bands contrast near the central part of the plate sliced from highly doped with As silicon crystal in the as-grown state. $\mathrm{MoK}_{\alpha}$ radiation, 440 reflection.

The important feature of section topograph structure is the presence of Kato interference fringes (the pendulum bands of Kato). But the bands are observed only in perfect, low-absorbing crystals, after [13] in such, for which macroscopic deformation of the crystal lattice $\Delta d / d$ do not exceed $10^{-6}$ ( $d$ is the interplanar distance).

Virtually on all the obtained section topographs, the pendulum bands of Kato were observed but with decreasing contrast in comparison with the perfect nondoped silicon samples of the same thickness. But on the certain topographs, the pendulum bands missed contrast gradually along the length of topographs. The length of topographs corresponded to the direction along the diameter of the silicon plates from the lateral surface to the ingot center. This is direction, along which it is possible to observe inhomogeneous distribution of impurities and defects. Fig. 1 shows a fragment of a typical topograph. The effect of the contrast missing was more clearly pronounced for the samples whose positions in the ingot corresponded to a higher oxygen concentration (in the vicinity of the seed and at the center of ingot, rather than at the periphery). By this means, the section topographs pointed to that in spite of the absence of dislocations the structure of these crystals was not perfect and was impaired in the direction from lateral surface to the ingot center. For the next study of the samples from central ingot part the diffractometry method based on Borrmann effect was employed [14].

The results of XRD measurements using 220 reflection in the conditions of $\mathrm{CuK}_{\alpha}$-radiation and $\mu t=17 \quad(\mu$ is the linear coefficient of photoelectric absorption and $t$ is the sample thickness) in the Laue geometry showed a decrease in the peak intensity (Borrmann effect suppression). The integral intensity of anomalous transmission is highly sensitive to small concentrations of defects in crystals, especially in cases where defects are clusters or small dislocation loops [15]. An increase in the X-ray absorption is caused by the displacement of atoms to the space between atomic diffraction planes. From the measurements of the integral intensity of anomalous X-ray transmission, the effective absorption coefficient can be evaluated. On the base of the dynamic theory in the absence of diffuse scattering in the crystals containing point defects, its value may be expressed via the effective static DebyeWaller factor $L$. This factor is a measure of average lattice distortion and can change in a wide range. For the samples in which the contrast missing of pendulum bands was observed, a correlation of the experimental and simulated values was achieved at $L \sim 0.02$. Because this value is much greater than that obtained for the uniform distribution of As in $\mathrm{Si}\left(L \sim 10^{-6}\right)$ in [10], it was concluded that the samples contain some clusters, for which the volume change per cluster is greater than the average volume change per As atom. These clusters most probably represent assemblies of As atoms, which do not form a new phase precipitate yet.

From the correlation of X-ray topographic and diffractometric data in [10], it was concluded that the phenomenon of cluster formation in the as-grown state of CZ-Si crystals highly doped with As takes place. The cluster formation during crystal growth in presence of As is more pronounced in the regions enriched with the growth-related impurity (oxygen). But in this case, the size of defects is probably very small for resolving their images on the section topographs, and their concentration is very low for observation using electron microscopy and other methods.

Using these means, in $[2,9,10]$ it was proposed to apply the combination of the section Lang method with X-ray diffractometry based on the Borrmann effect for diagnostics of silicon single crystals highly doped with As in the as-grown state. Hereafter, the experience of this study was spread on the silicon crystals highly doped with phosphorus and boron.

It turned out that the phenomenon of cluster formation in the as-grown state takes place in these crystals, too, but this process is much less pronounced and depends on the impurity type and concentration. For instance, in the crystals doped with $\mathrm{P}$ up to $1.1 \times 10^{20} \mathrm{~cm}^{-3}$ the pendulum bands on the section topographs were observed, the Borrmann effect suppression was not found. On the section topographs, the image of a certain "macrodefect" was revealed which is shown in Fig. 2. This "defect" neither yielded a kinematic image nor could be revealed by the projection topography techniques, but it still perturbed the interference bands on the section topographs.

The size of this "defect" was estimated at $0.25 \mathrm{~mm}$. The image of it was similar to that of defects observed in intentionally non-doped dislocation-free CZ-Si crystal [7], which contained dissolved oxygen at the concentration $7 \times 10^{17} \mathrm{~cm}^{-3}$ and was subjected to a multistage post-growth heat treatment. The size of defects observed in [7] reached $1 \mathrm{~cm}$ and they were 
interpreted as stable fluctuations of the concentration of microdefects (dispersed oxygen-containing precipitates). But the "defect" in our sample was several times smaller in size, induced much weaker distortions in the interference bands without a breaks of its and corresponded to a more perfect lattice than in the surrounding regions. Therefore, the nature of "defect" in our samples could be different from that of the defects reported in [7]. We suppose the phenomenon of cluster formation was not fully realized in the region of this "defect", and this region remained more perfect than the surrounded ones. In this case, the name "defect" is not correct, but the presence of it in the samples points on that the surrounded regions of the crystal are not perfect. We have to admit that silicon highly doped with phosphorus is inherently susceptible to cluster formation, albeit on a precursor level, to which the Borrmann effect is insensitive. These results can suggest that the sensitivity of the section topography technique with respect to the influence of statistically distributed microdefects on the interference bands contrast is higher than it is now commonly accepted. This sensitivity can be estimated across $L<0.02$ and $\Delta d / d \sim 10^{-5}$.

After [2, 9, 10], the combination of the section topography with X-ray diffractometry based on the Borrmann effect was applied to the silicon crystals highly doped with boron and subjected to technological annealings.

Study of silicon crystals highly doped with boron after technological annealings

After annealing under conditions $\mathrm{A}$ and $\mathrm{C}$, formation of microdefects was observed using all the techniques of $\mathrm{X}$ ray topography, including the section method (Fig. 3, $\mathrm{a}-\mathrm{c})$. It was so well expressed that the section topographs could be received in the strong reflections of 220 type, when they are narrow and received with a short exposure ( $\sim 2 \mathrm{~h})$. In Fig. 3, the series of topographs is shown for the samples placed near the lateral surface

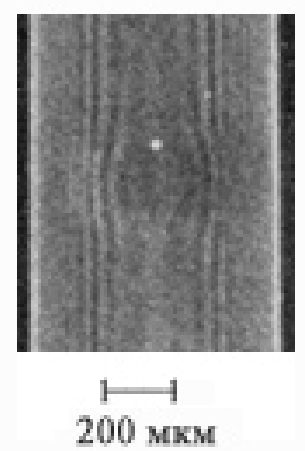

Fig. 2. X-ray diffraction image of a "macrodefect" on a section topograph of the highly doped with phosphorus silicon crystal in the as-grown state. $\mathrm{MoK}_{\alpha}$ radiation, 440 reflection. of a silicon ingot subjected to the heat treatment in the A mode. The projection topograph, in which a freemicrodefect zone is observed near the lateral surface of a silicon ingot, is shown on the left (a). The region of relatively rare defects is shown higher, and a combination of fine defects of a large density with rare large defects is shown at the top. A series of the section topographs obtained for the same sample as the projection topograph is shown on the right $(b, c)$. Section topographs sequentially correspond to the projection topograph's height. It is seen that the main details of the Kato bands remain in the center of section topographs in their width and at the edges under A mode, in spite of the image overlap of defects. The images of fine defects are not resolved on the topographs. The size of these defects can be of the order of $1 \mu \mathrm{m}$ and less. However, there are also large defects with the resolved images corresponding to dislocation loops. For comparison, in Fig. $3 \mathrm{~d}$ the section topograph of the sample not subjected to heat treatment (in the as-grown state) is shown. A distinction of the section topographs for the samples subjected to heat treatment in the $\mathrm{A}$ and $\mathrm{C}$ modes was mainly expressed in different visibility of the Kato bands. For this sign, the samples subjected to heat treatment in the $\mathrm{C}$ mode had a more damaged structure than in A mode.

Information on the real structure of the samples obtained by this method had a qualitative character; it was confirmed by the quantitative estimations based on the analysis of X-ray diffractometry data.

The data for defects were obtained from the analysis of the integral diffuse scattering that was measured in the thin crystal approximation on the tails of two-crystal rocking curves in 220 reflection [16-18]. The treatments of these curves after [17] allowed estimate concentration, distribution and the size of defects supposing that dislocation loops make the main contribution to X-ray scattering. For the samples annealed with respect to the A mode, the concentration of dislocation loops with the size $0.7-1.0 \mu \mathrm{m}$ reached the value $3 \times 10^{9} \mathrm{~cm}^{-3}$, and with respect to the $\mathrm{C}$ mode $1 \times 10^{11} \mathrm{~cm}^{-3}$ with the size $0.3-0.4 \mu \mathrm{m}$. The size and concentration of defects changed both along the ingot axis and along the diameter. As a rule, the defect density was notably smaller at the plate periphery, and the sizes of defects were larger. These data correspond to conclusions obtained on the basis of the X-ray topograph analysis.

The results obtained in this work allowed definite certain choice of two heat treatment modes for borondoped silicon from the viewpoint of the formation of microdefects. As a whole, the combination of X-ray diffraction methods, section Lang method and Borrmann effect diffractometry proved to be effective for diagnostics of highly doped silicon crystals both in the as-grown state and after high-temperature heat treatment. 


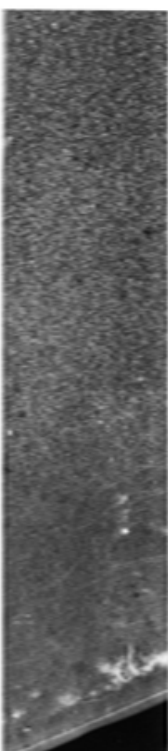

a

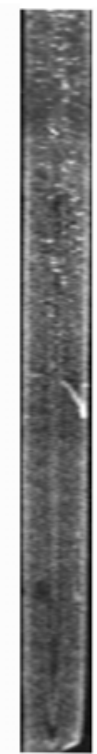

b

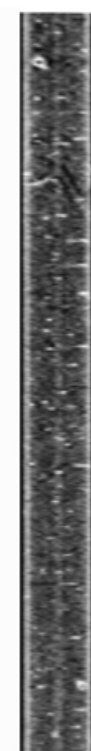

C

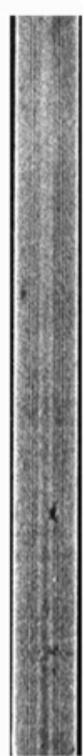

d
M 1:15

M 1:30

Fig. 3. Fragments of projection (a) and section (b, c, d) topographs of the highly doped with boron silicon crystal after heat treatment in the $\mathrm{A}$ mode $(\mathrm{a}-\mathrm{c})$ and in the as-grown state (d). $\mathrm{MoK}_{\alpha}$ radiation, 220 reflection.

\subsection{Research of strongly absorbing $\mathrm{GaSb}(\mathrm{Si})$ single crystals}

Study of almost all $\mathrm{A}_{3} \mathrm{~B}_{5}$ compounds single crystals virtually is not possible with the section Lang method for a reason of high X-ray absorption level. At the value $\mu t / \cos \theta>3$ (where $\theta$ is the Bragg angle) the Kato bands do not arise in the section topographs.

For crystals strongly absorbing X-rays, in particular, GaSb, the corresponding diagnostics can be performed on the basis of the topographic method of anomalous X-ray transmission using the rule described, for example, in [19]. It consists in that the dependence of the growth striations image's contrast on the direction of the diffraction vector indicates the absence of secondphase precipitates in them and the solid-solution state of the impurity. If there are precipitates in the growth striations, then the band images must be in all reflections, including those whose diffraction vector is perpendicular to the direction of crystal growth.

At the absence of precipitates, the straight-linear growth striations in these reflections virtually are not revealed because the change of interplanar distance for the planes parallel to growth direction is closed to zero by analogy with epitaxial systems [20].

However, this rule requires some confirmation because it is not clear whether it acts in the case when the precipitates of the second phase are not yet form fully or when they are coherent with matrix. So long as the data about the state of possible precipitates in
$\mathrm{GaSb}(\mathrm{Si})$ crystals are absent, the topographic research was supplemented with digital processing of the growth striation images, measurement of X-ray diffuse scattering, precise measurement of the lattice parameters and some electrical measurements.

If $\mathrm{MoK}_{\alpha}$ radiation was used, the sample thickness of $450 \mu \mathrm{m}$ provided $\mu t \sim 11$, which corresponded to the conditions used in the anomalous X-ray transmission method. Using this method, we obtained topographs in the symmetric 220 and 111 reflections as well as in the asymmetric 022 reflection; crystals and photoplates were scanned in one case and not scanned in the other case where a broad beam was used (the Barth-Hosemann method [21]). Recording in a set of Bragg reflections in $\mathrm{MoK}_{\alpha}$ radiation was used as a supplementary investigation technique.

Topographs of longitudinal plates (Figs 4a and $4 \mathrm{~b}$ ) are images of the seed and regrown part (RP) of the crystal. They are separated by a boundary reflecting the shape of the primary recrystallization front. It is weakly concave toward the seed. The comparison of the topographs of samples $\mathrm{N}$ and $\mathrm{K}$ shows:

- the images of growth striations in the seeds and the absence of them in the RPs;

- $\quad$ the single inclusions (or precipitates) of the second phase in the sample $\mathrm{N}$ and the absence of them in the sample K;

- the different number and distribution of dislocations in the regions of the seeds and RPs.

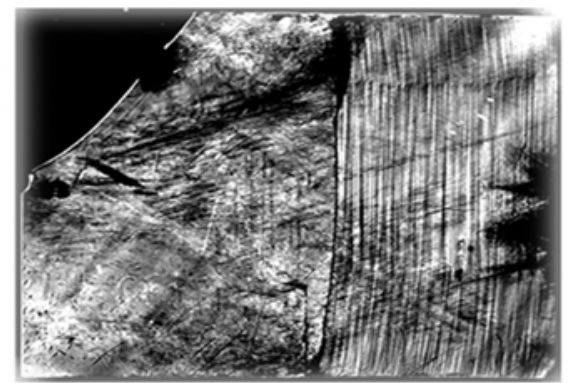

a

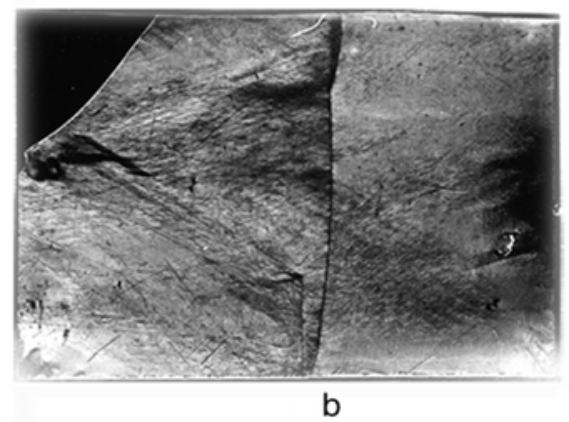

M 1:2

Fig. 4. X-ray topographs of longitudinal plates sliced from K$\mathrm{GaSb}(\mathrm{Si})$ crystal near the regrown boundary obtained in the symmetric 220 reflection with a good contrast of the growth striations in the seed (to the right) (a) and in the asymmetric 022 reflection (b) with an absence of its contrast. The method of anomalous X-ray transmission, $\mathrm{MoK}_{\alpha}$ radiation.

\section{(C) 2011, V. Lashkaryov Institute of Semiconductor Physics, National Academy of Sciences of Ukraine}


It is well known that the growth striations (the manifestation of a microsegregation inhomogeneity) are typical of highly doped crystals grown by the Czochralski method. The absence of growth striations in the RPs is related to the conditions of the stationary regime of crystal growth for strongly weakened thermogravitational convection in the melt. Therefore, we are mainly interested in the specific features of the growth band's images in the seeds.

Manifestation of the facet effect is clearly seen on the topograph (Fig. 4a) in the region of the seed. For most part of the seed, virtually straight-linear growth striations with the period of $\sim 43 \mu \mathrm{m}$ are typical. The images of growth striations on the topographs of both samples $(\mathrm{K}$ and $\mathrm{N})$ in the symmetric 220 reflections are practically identical. The difference is clearly seen in the asymmetric 022 reflection. Indeed, on the topograph of the sample $\mathrm{K}$ in the asymmetric 022 reflection, for which the direction of the diffraction vector $\mathbf{g}$ is perpendicular to the direction of the crystal growth $\mathbf{n}$, i.e., $(\mathbf{g} \cdot \mathbf{n})=0$, the images of growth striations were absent (Fig. 4b). On the topograph of the sample $\mathrm{N}$, they were not partially observed, only roughly in the center part of the seed by its length (region I, Fig. 2 in [22]). Thus, it can be deduced that there were indications of decomposition of solid solution of dopant in some regions of the seed of the sample $\mathrm{N}$ and the absence of those indications in the sample K.

Some quantitative characteristics of dopant microsegregation in crystals can be obtained by digital processing of the X-ray topography images of growth striations. Digital processing of obtained images consist in representation of the averaged brightness of growth striation images in the form of functional dependence, separation and the spectral Fourier analysis of the useful signal. In particular, the periodicity of the highestfrequency signal component is $\sim 43 \mu \mathrm{m}$, which is identical to the period of growth striations in the topographs of the seeds of the N (Fig. 5) and K crystals. It practically does not varied during translation along the crystals and characterizes the well-known periodic segregation effect associated with the crystal rotation during the growth when using the Czochralski method. The low-frequency region of the spectrum with several additional periodicities is an evidence of action of several mechanisms providing formation of concentration inhomogeneities and strongly changes along the crystal length. It probably characterizes smooth variations in the concentration and thermal fields in the vicinity of crystallization front because of the turbulent character of flows in the melt. The specific features of digital processing of growth striation images were considered in more detail in [23-25].

The topographic research was supplemented with precise measurements of the lattice parameters, with diffractometry studies, and with electro-physical measurements. The measurements of the lattice parameters in the different regions of the crystals demonstrated the inhomogeneity of dopant distribution and the distinction of its state in the samples $\mathrm{K}$ and $\mathrm{N}$.

The spectral method shows that the dopant concentration in the crystals under study is higher a little than the carrier density measured by the Hall method [25]. This means that the part of silicon is electrically inactive. Distribution of the charge carrier concentration in the seed is essentially more non-

\section{Region 1}
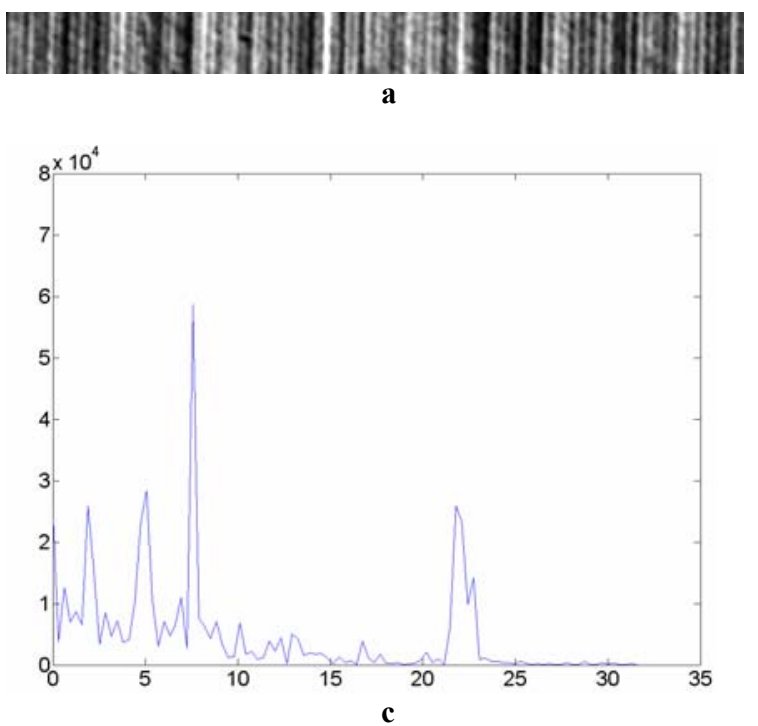

Region 2
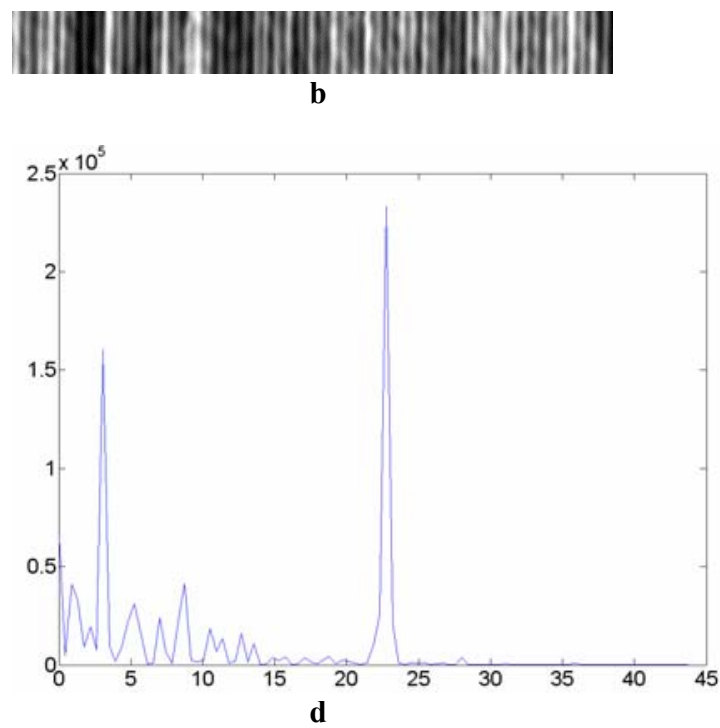

Fig. 5. Digital processing the growth striation images in the $\mathrm{GaSb}(\mathrm{Si})$ single crystals: $\mathrm{a}, \mathrm{b}$ - fragments of topographs of regions 1 $(\sim 10 \mathrm{~mm}$ from the regrown boundary, maximum concentration of charge carriers) and $2(\sim 3 \mathrm{~mm}$ from the regrown boundary, minimum concentration of charge carriers) of the seed of the $\mathrm{N}$ crystal, obtained in the symmetric 220 reflection; c, $\mathrm{d}-$ corresponding spectral densities of the distribution of the image brightness. $A$ - Fourier amplitude in arbitrary units, $f$-spatial frequency. 
uniform than in the regrown part. It should be noted that in the central part of the seed for the sample $\mathrm{N}$, where the growth striations in the 022 reflection are not revealed, the concentration of charge carriers measured by the Hall method is higher by approximately $20 \%$ than that in regions with explicitly expressed growth striations. This dependence can be related to the decay of the supersaturated solid solution in these regions and to the transformation of a portion of the dopant into the electrically inactive state. Charge carrier mobility in the regrown parts of $\mathrm{N}$ and $\mathrm{K}$ crystals noticeably exceeds the mobility in the seeds but there is macro-heterogeneity of its distribution both along the seeds and regrown parts. For the crystal $\mathrm{K}$ the difference in the charge carrier concentration between the seed and the RP is sharply pronounced, charge carrier mobility in the regrown part is considerably higher than in the seed and the RP of the crystal N. But the charge carrier concentration is uniformly distributed along the seed.

Thus, the electro-physical measurements have proved not only the difference in dopant state for $\mathrm{N}$ and $\mathrm{K}$ crystals, but also more uniform distribution of $\mathrm{Si}$ in the seed of the K crystal as compared to $\mathrm{N}$ crystal.

The study of the growth striations by diffractometry methods showed that they were of integral character and did not characterize individual bands, because the size of the X-ray beam $(\sim 100 \mu \mathrm{m})$ was relatively large and exceed the size of the growth striations $(\sim 43 \mu \mathrm{m})$. However, X-ray diffractometry demonstrated a high sensitivity to the level of microdistortions produced in the different stages of the decay of $\mathrm{Si}$ and $\mathrm{GaSb}$ supersaturated solid solution and provided valuable information about the structural state of the impurity.

Diffuse scattering measurements have proved to be particularly effective. Diffuse scattering (DS) was measured using a scheme with a rotating analyzer for the fixed detuning $\eta$ of the sample from the site of the reciprocal lattice. The following results were obtained $[25,26]$ :

a) DS is located in the vicinity of the site of the reciprocal lattice. Hence, it is caused by not separate microdefects (interstitial dopant atoms or dopant atoms at sites, vacancies, for which DS is located far from diffuse peak), but by the conglomerations of defects (clusters). However, the density of clusters and their size are small enough.

b) Higher intensity of DS on the one (small-angle) side of the rocking curve indicates that the aggregates of defects of the incorporated nature largely result in the registered DS (it probably corresponds to the sum of scattering from microdefects of incorporated and vacancy nature).

c) DS is sensitive to the non-uniform distribution of defects and correlates with the Hall curve along the seed: in the region of $10 \mathrm{~mm}$ near to the recrystallization boundary it is minimal. d) In the RP, an increase of the DS is noticeable, which reflects the process of increasing the concentration of interstitial $\mathrm{Si}$ and its clusters (small precipitates) because of both decrease of $\mathrm{Si}$ at sites (Hall method) and dissolution of large precipitates in the seed, which is noticeable on the topographs. According to the Hall method measurements the ratio of concentrations of $\mathrm{Si}$ at sites and interstitial $\mathrm{Si}$ is reduced from 2.5 in the seed to 1.3 in the RP of the sample.

Thus, the DS measurements allowed to confirm the previously obtained results and conclusions as well as allowed to receive additional information on the dopant state in the different parts of the studied crystals.

\section{Discussion}

The present work shows that diagnostics of the dopant state in highly doped crystals with various levels of Xray absorption is possible on the basis of different method combination. The section Lang method is most sensitive one, because it allows to notice the beginning of the solid solution decay process in the shape of formation of point defect clusters. But this is information only of quantitative character. For qualitative information, it was important to combine it with the Borrmann effect based X-ray diffractometry. This combination turned out is more useful for the quantitative estimation of a sensitivity of the section method expression via the Debye-Waller factor value.

It is known that the presence of the Kato bands on the section topographs is universally recognized and virtually only test on a structural perfection of the single crystals. Now this test received quantitative expression via the value of the static Debye-Waller factor $L \sim 0.02$ and macroscopic deformation of crystal lattice $\Delta d / d \sim 10^{-5}$ for the crystals containing clusters of the point defects. It is very of interest to note that this value of $L$ practically coincides with $L$ for non-doped silicon crystals containing dislocations with the density $5 \times 10^{3} \mathrm{~cm}^{-2}$ [27], for which the absence of the Kato bands on the section topographs was also observed [7]. Therefore, this value of $L$ can regard to be as some measure of a sensitivity of presence of the Kato bands on section topographs to crystal lattice distortions both by the point defects and dislocations. As to diagnostics of the strong-absorbing crystals, for them the role of the section method can be played by the method of X-ray anomalous transmission that needs the supplement of other techniques. The digital processing of the growth striation images enlarges the possibilities of this method.

\section{Conclusion}

1. The characterization of the dopant state in highly doped slight- and strong-absorbing crystals is important to be carried out on the basis of different methods. 
2. Application of the section X-ray topography in combination with the Borrmann effect based X-ray diffractometry is most effective for the slightly absorbing crystals. With regard to the crystals in the asgrown state, only these methods are the most effective ones, because they allow to notice the early stage of a decay of impurity solid solution.

3. The method of X-ray anomalous transmission together with measurements of X-ray diffuse scattering and electro-physical methods is most effective for the study of strongly absorbing crystals. The digital processing technique enlarges possibilities of the X-ray topographic method.

4. For diagnostics of highly doped crystals after a post-growth annealing, it is possible to use the standard techniques, but the above mentioned ones provide supplement information about distribution, concentration and size of defects. Using them, a substantial choice of the best annealing is possible with a viewpoint of microdefect formation.

\section{Acknowledgement}

The work was supported by the Russian Foundation for Basic Research, project no. 06-02-1624 and by the Russian Foundation for Basic Research and the Government of Kaluga region, project no. 09-02-97516.

\section{References}

1. V.I. Fistul', Highly Doped Semicoductors. Nauka, Noscow, 1967 (in Russian).

2. S.S. Rouvimov, R.N. Kyutt, J. Kearns et al., Defect formation in heavily As-doped CZ Si // Solid State Phenomena 95-96, p. 17-22 (2004).

3. M.G. Mil'vidskii, A.V. Kartavikh, V.V. Rakov, Growth of single crystals from the melt in spacecraft conditions // Surface investigation. $X$ ray, Synchrotron and Neutron Techniques, 9, p. 17 35 (2001), in Russian.

4. Yu.A. Serebryakov, I.A. Prokhorov, V.N. Vlasov et al., Concentration and structure inhomogeneities in $\mathrm{GaSb}(\mathrm{Si})$ single crystals grown at different heat and mass transfer conditions // J. Cryst. Growth, 304, p. 11-21 (2007).

5. A.A. Lomov, P. Zaumseil, U. Winter, Characterization of process-induced defects in silicon with triple-crystal diffractometry // Acta Cryst. A41, p. 223-227 (1985).

6. M. Lefeld-Sosnowska, J. Grokowski, G. Kowalski, A study of defects generated in Czochralski-grown Si during two-step annealing // J. Phys. D: Appl. Phys. 28, p. A42-A46 (1985).

7. M.G. Mil'vidskii, Yu.A. Osip'yan, I.A. Smirnova et al., Observation of macrodefects in silicon by the methods of X-ray topography // Surface investigation. X-ray, Synchrotron and Neutron Techniques, 6, p. 5-10 (2001), in Russian.
8. P. Klang, V. Holy, J. Kubena et al., X-ray diffuse scattering from defects in nitrogen-doped Czochralski grown silicon wafers // J. Phys. D: Appl. Phys. 38, p. A105-A110 (2005).

9. R.N. Kyutt, I.L. Shulpina, G.N. Mosina et al., Xray diffraction study of defect distribution in Czochralski grown silicon highly doped by As // J. Phys. D: Appl. Phys. 38, p. A111-A116 (2005).

10. R.N. Kyutt, S.S. Rouvimov, I.L. Shul'pina, Diagnostics of highly doped silicon single crystals grown by Czochralski method // Pis'ma zhurnal tekhnich. fiziki, 32(24), p. 79-84 (2006), in Russian.

11. D.K. Bowen, B.K. Tanner, High Resolution X-ray Diffractometry and Topography. London, Taylor and Francis, 1998.

12. P.F. Fewster, N.L. Andrew, Absolute latticeparameter measurement // J. Appl. Cryst. 28, p. 451-458 (1995).

13. I.1. Shul'pina, Section methods of X-ray topography and its possibilities in basic research and application // Zavodskaya laboratoria. Diagnostika materialov, 73(5), p. 30-35 (2007), in Russian.

14. J. Barushel, J. Hartwig, Basic courses of X-TOP 2002. Grenoble, France, 2002, p. 1-38.

15. R.N. Kyutt, X-ray Bragg diffraction in crystals with dislocations // Crystallography, 33(4), p. 827-830 (1988), in Russian.

16. P.H. Dederichs, The theory of diffuse X-ray scattering and its application to the study of point defects and their clasters // J. Phys. F: Met. Phys. 3, p. 471-495 (1973).

17. B.C. Larson, X-ray studies of defect clusters in copper // J. Appl. Cryst. 8, p. 150-156 (1975).

18. I.L. Shul'pina, S.S. Rouvimov, R.N. Kyutt, X-ray diffraction study of silicon single crystals highly doped by boron // Surface investigation. X-ray, Synchrotron and Neutron Techniques, 1, p. 38-42 (2010), in Russian.

19. G.H. Schwuttke, Direct observation of imperfections in semiconductor crystals by anomalous transmission of X-rays // J. Appl. Phys. 33, p. 2760-2767 (1962).

20. V.A. Bushuev, R.N. Kyutt, Yu.P. Khapachev, Physical Principles of X-ray Diffractometry Characterization of Real Structure Parameters in Multilayer Epitaxial Films. Nal'chik, 1996 (in Russian).

21. H. Barth, R. Hosemann, Anwendung der Parallelstrahlmethodeim Durchstrahlungsfall zur Prufung der Kristallinneren mit Rontgen-Strahlen // Zs. Naturforsch. B.13a, p. 792 (1958).

22. I.A. Prokhorov, Yu.A. Serebryakov, E.N. Korobeinikova et al., X-ray study of structural features of $\mathrm{GaSb}(\mathrm{Si})$ single crystals grown using a set of diffraction methods under various heat and mass transfer conditions // Surface investigation. $X$ ray, Synchrotron and Neutron Techniques, 1(3), p. 255-259 (2007). 
23. I.A. Prokhorov, I.Zh. Bezbakh, B.G. Zakharov et al. X-ray topography study of microsegregation in crystals // Surface investigation. X-ray, Synchrotron and Neutron Techniques, 1(3), p. 260264 (2007).

24. I.A Prokhorov, Yu.A. Serebryakov, B.G. Zakharov et al., Growth striations and dislocations in highly doped semiconductor single crystals // J. Cryst. Growth, 310(24), p. 5477-5482 (2008).

25. I.A. Prokhorov, Yu.A. Serebryakov, I.Zh. Bezbakh et al., Concentration and structural inhomogeneities in highly doped $\mathrm{GaSb}(\mathrm{Si})$ single crystals // Surface investigation. X-ray, Synchrotron and Neutron Techniques, 3(6), p. 936-942 (2009).

26. I.L. Shul'pina, R.N. Kyutt, V.V. Ratnikov I.A. Prokhorov, I.Zh. Bezbakh, M.P. Shcheglov, $\mathrm{X}$-ray diffraction diagnostics methods as applied to highly doped semiconductor single crystals // Zhurnal tekhnich fiziki, 80(4), p. 105-114 (2010), in Russian.

27. L.I. Datsenko, V.B. Molodkin, M.E. Osinovskii, Dynamic X-ray Scattering by the Real Crystals. Naukova dumka, Kiev, 1988 (in Russian). 